\title{
A Short Note on Non-isothermal Diffusion Models
}

\section{T. Ficker}

Asymptotic behaviour of the DIAL and DRAL non-isothermal models, derived previously for the diffusion of water vapour through a porous building structure, is studied under the assumption that the initially non-isothermal structure becomes purely isothermal.

Keywords: Isothermal and non-isothermal diffusions, diffusion models, condensation in building structures.

\section{Introduction}

In a previous research communication [1], the DIAL and DRAL non-isothermal diffusion models were derived and compared with the standard isothermal models commonly used in building thermal technology. In a further related communication [2] we applied these models to the Glaser condensation scheme. The Glaser scheme enables, among others, an assessment of condensate for a one-year period. Naturally, throughout the year the structure is mostly exposed to non-isothermal states but, especially in the summer season, it may be subjected to a purely isothermal state. The DIAL and DRAL models provide the basic relations solely for non-isothermal conditions, i.e., they contain different temperatures $T_{1}, T_{2}$ belonging to the opposite sides of the structure. When approaching the isothermal state $\left(T_{2} \rightarrow T_{1}\right)$, the relations give an uncertain expression $0 / 0$ and, at first sight, it is not clear how these relations should be applied to the isothermal state $\left(T_{2}=T_{1}=T\right)$.

This short communication is aimed at deriving asymptotic DIAL and DRAL relations holding for the isothermal state of a building structure possessing the diffusion resistance factor $\mu$.

\section{Asymptotic DIAL relations}

For the DIAL model the generalised diffusion resistance $R_{d}^{*}$ and diffusion 'conductivity' $D_{\text {eff }}^{*}$ read [1]

$R_{\mathrm{eff}}^{*}=\frac{d}{D_{\mathrm{eff}}^{*}}, \quad D_{\mathrm{eff}}^{*}=\frac{(2-n) k p_{a}}{\mu R_{a}} \frac{T_{1}-T_{2}}{T_{1}^{2-n}-T_{2}^{2-n}}$,

$p_{a}=98066.5 \mathrm{~Pa}, k=8.9718 \cdot 10^{-10} \mathrm{~m}^{2} \mathrm{~s}^{-1} \mathrm{~K}^{-1.81}, n=1.81$.

To determine the relations describing the isothermal state $\left(T_{2}=T_{1}=T\right)$ it is necessary to use the l'Hospital rule

$$
\begin{aligned}
\lim _{T_{2} \rightarrow T_{1}} \frac{T_{1}-T_{2}}{T_{1}^{2-n}-T_{2}^{2-n}} & =\lim _{T_{2} \rightarrow T_{1}} \frac{\frac{d}{d T_{2}}\left(T_{1}-T_{2}\right)}{\frac{d}{d T_{2}}\left(T_{1}^{2-n}-T_{2}^{2-n}\right)}= \\
& =\frac{T_{2}^{n-1}}{2-n}=\frac{T^{n-1}}{2-n},
\end{aligned}
$$

which leads to the following result

$R_{\mathrm{eff}}^{*}=\frac{d}{D_{\mathrm{eff}}^{*}}, \quad D_{\mathrm{eff}}^{*}=\frac{k p_{a}}{\mu R_{a}} T^{n-1}=\frac{k p_{a}}{\mu R_{a}} T^{0.81}$.

\section{Asymptotic DRAL relations}

Repeating the same procedure as in the foregoing section, we can rewrite the original DRAL non-isothermal relations [1]

$R_{\text {eff }}=\frac{d}{D_{\text {eff }}}, D_{\text {eff }}=\frac{(n-1) k}{\mu} \frac{T_{1}-T_{2}}{T_{2}^{1-n}-T_{1}^{1-n}}$

using the l'Hospital rule

$$
\begin{aligned}
\lim _{T_{2} \rightarrow T_{1}} \frac{T_{1}-T_{2}}{T_{2}^{1-n}-T_{1}^{1-n}} & =\lim _{T_{2} \rightarrow T_{1}} \frac{\frac{d}{d T_{2}}\left(T_{1}-T_{2}\right)}{d T_{2}\left(T_{2}^{1-n}-T_{1}^{1-n}\right)}= \\
& =\frac{T_{2}^{n}}{n-1}=\frac{T^{n}}{n-1}
\end{aligned}
$$

into the purely isothermal relations $\left(T_{2}=T_{1}=T\right)$

$$
R_{\text {eff }}=\frac{d}{D_{\text {eff }}}, \quad D_{\text {eff }}=\frac{k}{\mu} T^{n}=\frac{k}{\mu} T^{1.81} .
$$

Relation (6) exactly corresponds to the isothermal IM-TDR result [1], which confirms the consistency of the developed models.

\section{Conclusion}

Derived asymptotic relations (3) and (6) represent a necessary complement for the DLAL and DRAL non-isothermal models when they are faced with the task of estimating the water condensate inside building structures exposed to purely isothermal conditions. Such problems may appear in building thermal technology when calculating the one-year balance of condensate inside the building envelopes.

\section{References}

[1] Ficker, T., Podešvová, Z.: Models for Non-Isothermal Steady-State Diffusion in Porous Building Materials. Acta Polytechnica (accepted for publication).

[2] Ficker, T., Podešvová, Z.: Modified Glaser's Condensation Model. Acta Polytechnica (accepted for publication).

Assoc. Prof. RNDr. Tomáš Ficker, DrSc.

phone: + 420541147661

e-mail: fyfic@fce.vutbr.cz

Department of Physics

University of Technology

Faculty of Civil Engineering

Žižkova 17

66237 Brno, Czech Republic 